\title{
Milk mid-infrared spectral data as a tool to predict feed intake in lactating Norwegian Red dairy cows
}

\author{
S. E. Wallén, ${ }^{*}$ E. Prestløkken, ${ }^{*}$ T. H. E. Meuwissen,${ }^{* 1}$ S. McParland, $†$ and D. P. Berry† \\ *Norwegian University of Life Sciences, Department of Animal and Aquacultural Sciences, Ås, 1432, Norway \\ †Animal and Grassland Research and Innovation Centre, Teagasc, Moorepark, Co. Cork, P61C996, Ireland
}

\begin{abstract}
Mid-infrared (MIR) spectroscopy of milk was used to predict dry matter intake (DMI) and net energy intake (NEI) in 160 lactating Norwegian Red dairy cows. A total of 857 observations were used in leave-one-out cross-validation and external validation to develop and validate prediction equations using 5 different models. Predictions were performed using (multiple) linear regression, partial least squares (PLS) regression, or best linear unbiased prediction (BLUP) methods. Linear regression was implemented using just milk yield (MY) or fat, protein, and lactose concentration in milk (Mcont) or using MY together with body weight (BW) as predictors of intake. The PLS and BLUP methods were implemented using just the MIR spectral information or using the MIR together with Mcont, MY, BW, or NEI from concentrate (NEIconc). When using BLUP, the MIR spectral wavelengths were always treated as random effects, whereas Mcont, MY, BW, and NEIconc were considered to be fixed effects. Accuracy of prediction $(\mathrm{R})$ was defined as the correlation between the predicted and observed feed intake testday records. When using the linear regression method, the greatest $\mathrm{R}$ of predicting DMI (0.54) and NEI (0.60) in the external validation was achieved when the model included both MY and BW. When using PLS, the greatest R of predicting DMI (0.54) and NEI (0.65) in the external validation data set was achieved when using both BW and MY as predictors in combination with the MIR spectra. When using BLUP, the greatest $\mathrm{R}$ of predicting DMI (0.54) in the external validation was when using MY together with the MIR spectra. The greatest $\mathrm{R}$ of predicting NEI (0.65) in the external validation using BLUP was achieved when the model included both BW and MY in combination with the
\end{abstract}

Received September 22, 2017.

Accepted February 22, 2018.

${ }^{1}$ Corresponding author: theo.meuwissen@nmbu.no
MIR spectra or when the model included both NEIconc and MY in combination with MIR spectra. However, although the linear regression coefficients of actual on predicted values for DMI and NEI were not different from unity when using PLS, they were less than unity for some of the models developed using BLUP. This study shows that MIR spectral data can be used to predict NEI as a measure of feed intake in Norwegian Red dairy cattle and that the accuracy is augmented if additional, often available data are also included in the prediction model.

Key words: mid-infrared spectroscopy, dry matter intake, net energy intake, prediction

\section{INTRODUCTION}

Dairy cattle breeding goals have advanced from being traditionally narrow in focus to now being more holistic and including functional traits (Miglior et al., 2005). In the Nordic countries, health and fertility traits have been included in the breeding goal since the 1970s (Philipsson and Lindhé, 2003). Traits not currently explicitly included in most dairy cow breeding goals include feed intake, product quality, and the environmental footprint (Berry, 2015). Breeding goal traits must be economically important, must exhibit genetic variation, and should ideally be (easily) measured at a low cost in a large population of animals or, at least, should be genetically correlated with heritable traits that can be routinely measured. Improving feed efficiency is economically important because feed is the major single variable cost in dairy production (Shalloo et al., 2004). Genetic variability in feed intake (and efficiency) in dairy cows is known to exist (Svendsen et al., 1993; Berry and Crowley, 2013; Hurley et al., 2017). Thus, the main factor hampering the inclusion of feed intake directly in a breeding objective is routine access to phenotypic data of feed intake from a large population of animals, ideally at a low cost, to achieve a high accuracy of selection.

McParland et al. (2014) documented that feed intake in lactating dairy cows could be predicted using mid- 
infrared (MIR) spectrometry of milk. Mid-infrared spectroscopy is based on the study of the interaction between matter and electromagnetic waves (De Marchi et al., 2014) in the 900 to $5,000 \mathrm{~cm}^{-1}$ region and is routinely used globally to determine fat, protein, and lactose concentration in milk (Soyeurt et al., 2011; De Marchi et al., 2014). Because individual animal milk samples are routinely taken as part of day-to-day dairy herd management, using these samples to also predict feed intake would be a cost-effective strategy for generating data for management purposes as well as for inclusion in a breeding program. McParland et al. (2014), however, evaluated only the prediction of feed intake in lactating Holstein-Friesian dairy cows. Energy intake, energy balance, and residual energy intake have been successfully predicted using MIR in 2 production systems in the United Kingdom and Ireland (McParland and Berry, 2016). In addition, we (unpublished data) used pooled MIR data from Irish Holstein-Friesian and Norwegian Red dairy cattle to predict net energy intake (NEI) and effective energy intake and concluded that exploiting pooled MIR data can further increase the accuracy of predicting energy intake-related traits. Traditionally, the partial least squares (PLS) regression method has mainly been used for developing the prediction equations in MIR studies (McParland et al., 2011, 2012, 2014; De Marchi et al., 2014), but recently Ferragina et al. (2015) proposed the use of genomic prediction approaches for MIR-based predictions. The objective of the present study was to determine whether MIR spectral data from milk are suitable for predicting feed intake in lactating Norwegian Red dairy cows. Also of interest was whether prediction accuracy could be improved using best linear unbiased prediction (BLUP) methodology, which is commonly used in genetic and genomic prediction, relative to PLS regression approaches, which have heretofore been the only approach used to predict feed intake from milk MIR data.

\section{MATERIALS AND METHODS}

\section{Data}

Data from 6 different feeding experiments were collected from the dairy research farm at the Norwegian University of Life Sciences (Ås, Norway) between 2007 and 2015, with the exception of 2010 and 2012, from which DMI data could not be recovered. Traits periodically available included individual cow DMI; milk yield (MY); fat, protein, and lactose concentration in milk (Mcont); and individual cow BW (Table 1). Data were available from 204 lactations from 160 Norwegian Red dairy cows; the total number of test-day records for each trait was 857 .

In all experiments, cows were fed timothy grass-based silage combined with grain-based concentrate (Table 2 ). The concentrate contained mainly barley and oats supplemented with rapeseed cake and soybean meal as the main protein source. The protein concentration of the feed varied from 12 and $20 \%$ of DM (Table 2). Concentrates were fed according to individual cow MY ( 4 of the 6 feed treatments) or in fixed quantities ( 2 of the 6 feed treatments) using automatic feed stations. In all instances, silage was either fed ad libitum or restricted using feed bins fitted with vertical feed gates and weighing cells underneath.

The DM of the silage was calculated based on the feed analyses. For concentrate, DM was based on feed analyses, or tabulated information in the feed tables from the Nordic feed evaluation system (NorFor; Volden, 2011). Individual feed intake and DM of feed were used to calculate the DMI of silage and concentrate separately. Daily energy intake, expressed as NEI, was calculated based on the NorFor evaluation system and is described in detail in the Appendix. Net energy intake was calculated from the DMI of silage and concentrate separately. Net energy intake for silage (MJ/kg of DM) was calculated based on the chemical composition of the feed using standard feed values in NorFor. If NEI for concentrate was missing, it was calculated using a default DM of $86 \%$ and an energy content of $7.3 \mathrm{MJ} / \mathrm{kg}$ of DM. Both DMI and NEI for silage and concentrate were summed to get total DMI and NEI per cow.

Predicted values for each performance trait were obtained from interpolation of the actual observations for Mcont, DMI, NEI, MY, and BW; cubic splines with 6 knot points at 20,70,120,170,220, and 270 DIM were fitted through individual test-day records of the traits to facilitate the interpolation. After interpolation, several restrictions were implemented to improve the

Table 1. Phenotypic records on the days with milk mid-infrared spectral information for 160 cows and 204 lactations

\begin{tabular}{lrc}
\hline Trait $^{1}$ & Mean & SD \\
\hline DMI (kg/d) & 19.83 & 3.52 \\
MY (kg/d) & 24.79 & 5.93 \\
Fat (\%) & 4.37 & 0.55 \\
Protein (\%) & 3.42 & 0.28 \\
Lactose (\%) & 4.65 & 0.18 \\
BW (kg) & 557.38 & 54.91 \\
NEI (MJ/d) & 125.58 & 22.00 \\
\hline
\end{tabular}

${ }^{1} \mathrm{MY}=$ milk yield; fat $=$ fat concentration in milk; protein $=$ protein concentration in milk; lactose $=$ lactose concentration in milk; $\mathrm{NEI}=$ net energy intake. 


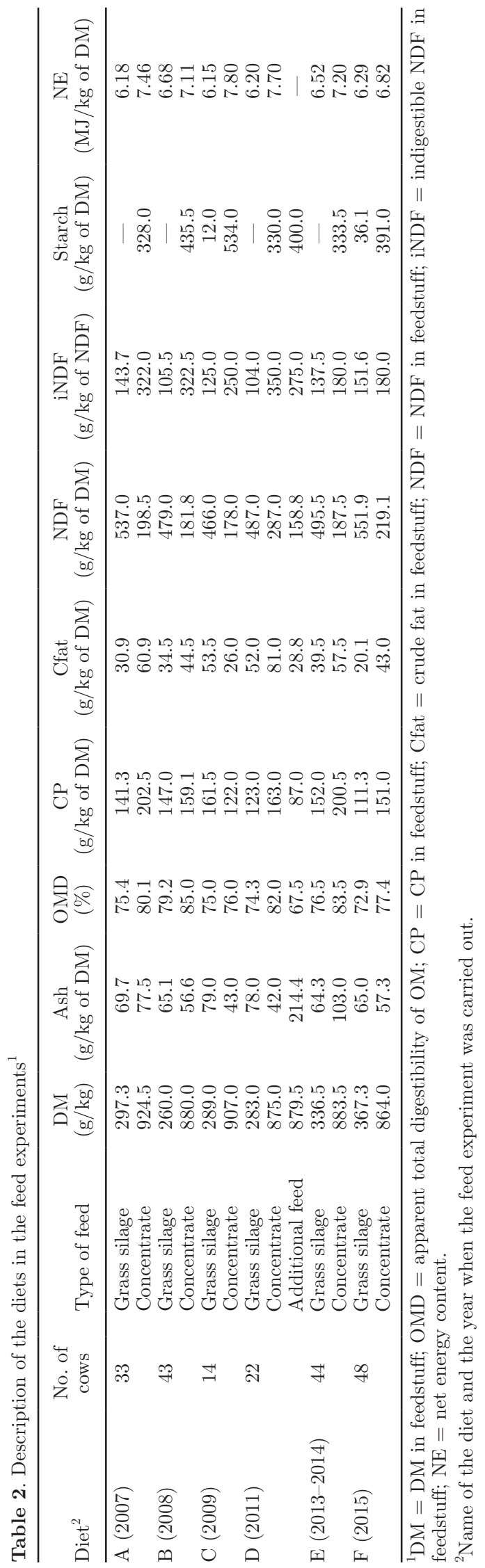

integrity of the data. Milk yield and milk composition records were discarded if MY was $<10 \mathrm{~kg}$. Records were also excluded if fat concentration in milk was $>6.5 \%$, total DMI was $>30 \mathrm{~kg}$, or BW was $<400 \mathrm{~kg}$. Net energy intake values $<55 \mathrm{MJ}$ or $>400 \mathrm{MJ}$ were also excluded from further analysis. A total of 15 data points (i.e., $1.7 \%$ of the data) were discarded during this data editing step.

\section{MIR Data}

Cows were milked twice daily between 0615 and 0900 $\mathrm{h}$ and again between 1500 and $1715 \mathrm{~h}$. Milk samples were collected with varying frequency for each of the different experiments (Table 3). All milk samples were conserved with bronopol (2-bromo-2-nitropropane-1,3diol) and then stored at $4{ }^{\circ} \mathrm{C}$. All milk samples were analyzed using the same MIR spectrometer (MilkoScan FT6000; Foss Electric A/S, Hillerød, Denmark), and the resulting spectra were stored. The absorption of infrared light through the milk sample at wavelengths in the 900 to $5,000 \mathrm{~cm}^{-1}$ region is represented by 1,060 data points in the Foss MIR spectrum. Mid-infrared wavelength regions known to be related to water absorbance were not considered in the analysis (Zimmermann and Kohler, 2013). Preliminary tests of alternative wavelength regions yielded the most suitable wavelength regions for each of the 2 traits. For NEI, the wavelength regions of 926 to 1,601, 1,701 to 1,805, and 2,693 to $3,069 \mathrm{~cm}^{-1}$, which reflect the protein and fat regions, were used. For DMI, the regions used were between 926 and 1,593, between 1,745 and 3,061, and between 3,781 and $5,149 \mathrm{~cm}^{-1}$.

In the experiments carried out between the years 2007 and 2014, spectral data were available only from a composite morning and evening milk sample. In the experiment undertaken in the year 2015, spectral data were taken separately from the morning and evening milkings. A weighted average of fat, protein, and lactose concentration as well as each spectrum wavelength was therefore calculated for data collected in the year 2015 , so the data from all years were comparable; a total of 473 daily records were available from the year 2015 where this approach was applied. Each wavelength value of the available 857 spectral records was scaled such that the mean and standard deviation of each of the wavelengths were 0 and 1 , respectively. As a separate treatment, Savitzky-Golay smoothing was applied to the untreated MIR spectral data to smooth a signal by fitting a polynomial to a sliding window of MIR data. Different degrees of polynomial and window sizes were tested using the Unscrambler X program (version 10.3, Camo Software AS, Oslo, Norway). 


\section{Development and Validation of Prediction Equations}

Prediction models were developed using leave-oneout cross-validation. In this approach, a single observation was left out, one by one, as a single-element test set while all the other observations were included in the calibration set. This was iterated until every sample had been predicted once. To obtain a more appropriate representation of the accuracy and robustness of the prediction equations, external validation was also performed. This was achieved by randomly stratifying animals into 5 external validation data sets, ensuring that all the records of a given animal appeared only in 1 validation set, so that the data of an animal were never present in both the calibration and validation data sets at the same time. Observations in each external validation data set were then predicted using the model developed from only the observations in the respective calibration data set (prediction models developed from data on the calibration animals were applied to predict the respective trait in the validation animals).

Prediction equations for DMI and NEI were developed and cross-validated using 9 different model constructs: (1) using only the observations of Mcont, (2) using only the observations of MY, (3) using the observations of both MY and Mcont, (4) using only the MIR spectral information, (5) using the observations of Mcont and the associated MIR spectral information, (6) using the observations of both MY and the associated MIR spectral information, (7) using the observations of both MY and the BW of the cow, (8) using the observations of both MY and the associated MIR spectral information as well as the BW of the cow, and (9) using the observations of both MY and the associated MIR spectral information as well as the energy intake from concentrate (NEIconc). Models 1 to 8 were used to predict both DMI and NEI, whereas model 9 was used to predict only NEI. The same data set was used for all model constructs and validation.

Models 1, 2, 3, and 7 were implemented using (multiple) linear regression (LR) models. For models 4, 5, 6,
8, and 9, 2 alternative statistical approaches were used to predict either NEI or DMI, namely PLS (PROC PLS; SAS Institute Inc., Cary, NC) or BLUP. Best linear unbiased prediction was implemented using the same approach as in genomic prediction except that marker loci were replaced by the wavelengths of the spectra (Meuwissen et al., 2001). In BLUP, the MIR spectra wavelengths were always treated as random effects; when fitting models 5, 6, 8, and 9, Mcont, MY, $\mathrm{BW}$, and NEIconc were always considered as fixed effects. Matlab (R2016a, The MathWorks Inc., Natick, MA) scripts were used to perform all BLUP analyses. The model for the BLUP analysis was

$$
\mathbf{y}=\mathbf{X b}+\mathbf{Z u}+\mathbf{e}
$$

where $\mathbf{y}$ is a vector of NEI or DMI records, which were scaled such that the mean and standard deviation of each trait were 0 and 1 , respectively; $\mathbf{b}$ is a vector of fixed effects containing an overall mean and, where appropriate, the additional effects of Mcont, MY, BW, and NEIconc scaled such that the mean and standard deviation of each trait was 0 and 1 , respectively; $\mathbf{X}$ is the design matrix containing columns of covariates for the effects in $\mathbf{b} ; \mathbf{Z}$ is a matrix of scaled MIR spectra; $\mathbf{u}$ is the random effect of the spectral wavelengths, with $\operatorname{Var}(\mathrm{u})=\mathbf{I} \sigma_{\mathrm{u}}{ }^{2}$, where $\mathbf{I}$ is an identity matrix and $\mathbf{e}$ is a vector of residuals with $\operatorname{Var}(\mathrm{e})=\mathbf{I} \sigma_{\mathrm{e}}{ }^{2}$. The variance components $\sigma_{\mathrm{u}}{ }^{2}$ and $\sigma_{\mathrm{e}}{ }^{2}$ are unknown, but solving the mixed model equations to obtain solutions for $\mathbf{b}$ and $\mathbf{u}$ only requires knowing the ratio of the variances $\lambda$ $=\sigma_{\mathrm{e}}{ }^{2} / \sigma_{\mathrm{u}}{ }^{2}$. Different values of $\lambda$ were tested, and the $\lambda$ value that resulted in the greatest accuracy of prediction in the external validation was chosen separately for each trait and model. The effect of different values of $\lambda$ on leave-one-out cross-validation was also considered.

The main difference between the BLUP and PLS approach is that the BLUP approach uses all eigencomponents of the milk spectra to predict NEI and DMI but reduces the weight of the smallest eigencomponents

Table 3. Summary of feeding experiments ${ }^{1}$

\begin{tabular}{llcrc}
\hline Experiment & Experiment period & $\begin{array}{c}\text { No. of } \\
\text { cows }\end{array}$ & $\begin{array}{c}\text { No. of } \\
\text { records }\end{array}$ & Frequency \\
\hline A & Jan. 1 to May 7, 2007 & 33 & 33 & 1.00 \\
B & Jan. 8 to Apr. 2, 2008 & 43 & 119 & 2.77 \\
C & Feb. 2 to Apr. 5, 2009 & 14 & 14 & 1.00 \\
D & Aug. 22 to Dec. 9, 2011 & 22 & 61 & 2.77 \\
E & Sep. 1, 2012, to Jan. 5, 2014 & 44 & 128 & 2.91 \\
F & Jan. 19 to Mar. 13, 2015 & 48 & 502 & 10.46 \\
\hline
\end{tabular}

${ }^{1}$ No. of cows $=$ total number of cows within each feeding experiment. No. of records $=$ total number of collected records for DMI; milk yield; fat, protein, and lactose concentration in milk; BW; and net energy intake within each feeding experiment. Frequency $=$ how frequently records were collected per cow and within each feeding experiment. 
proportionally more (which is determined by $\lambda$ in that $\lambda=0$ implies full weights for all components). The strong reduction of the weight on the smallest components may reflect the minimal information contributed by some wavelengths. Partial least squares restricts itself to the components associated with the dependent variable and ignores the small components altogether, whereas the biggest components obtain their full weight (the number of components with full weight is determined by cross-validation).

The square root of the coefficient of determination from the regression model of true on predicted values (i.e., the correlation between true and predicted values) was used as a measure of the accuracy $(\mathrm{R})$ of prediction. In the PLS regression analysis, a variable number of explanatory factors is used to explain the maximum amount of variation of the correlated wavelength values as well as their correlation with the dependent variable. Increasing the maximum number of explanatory factors permitted in the prediction models can improve the accuracy of cross-validation but may reduce the accuracy of prediction in external validation. Determining the maximum number of explanatory factors in the model was achieved by visually inspecting the changes in $\mathrm{R}$ for leave-one-out cross-validation and external validation. When undertaking the external validation of the PLS analysis, model performance was also assessed by the mean bias of prediction as well as both the root mean squared error of prediction and the LR coefficient (b) of true values on their respective predicted values using simple least squares regression.

\section{RESULTS}

The mean phenotypic values for the different performance traits of the 160 cows, on days where MIR data were also available, are summarized in Table 1. Average daily DMI and NEI were $19.8 \mathrm{~kg}$ and $125.6 \mathrm{MJ}$, respectively. The average NEI and DMI lactation profiles on days with MIR spectral data are in Figures 1 and 2. When using the LR models without MIR spectral information (i.e., models 1-3 and 7), the prediction accuracy in external validation ranged from 0.10 to 0.54 for DMI and from 0.25 to 0.60 for NEI (Tables 4 and 5). Of all the LR models, the greatest $\mathrm{R}$ of predicting both DMI and NEI in the external validation was achieved when the LR model included both MY and BW. The prediction accuracy was lower in the external validation compared with the leave-one-out cross-validation when using the LR models to predict DMI and NEI. For all the LR models, the average LR coefficient of actual on predicted NEI and DMI in the external validation was not different from $1(P>0.05)$. In addition, the mean bias for NEI and DMI in the external validation for these models was not different from $0(P>0.05)$, indicating unbiased prediction. Relative to using untreated spectral data, using smoothed MIR wavelengths did not improve the prediction accuracies; reported results

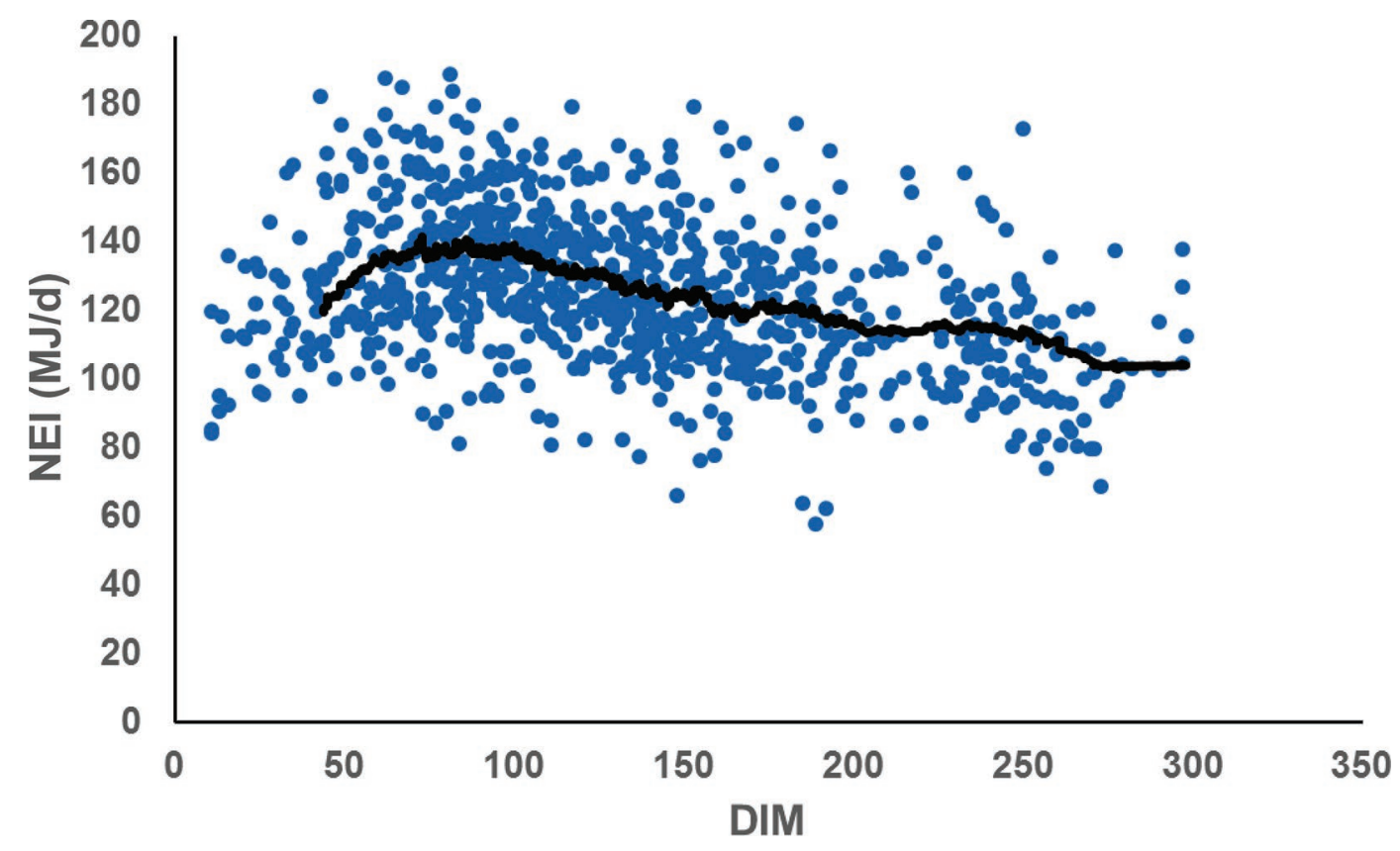

Figure 1. Lactation profile of the net energy intake (NEI) on days with milk mid-infrared spectral recordings with an average trend line. Color version available online. 
Table 4. Average number of factors (Fac), root mean squared error (RMSE), correlation coefficient (r), mean bias (SE in parentheses), ${ }^{1}$ and slope (b; SE in parentheses) ${ }^{2}$ obtained from predicting DMI using centered and scaled mid-infrared (MIR) spectra, tested using leave-one-out cross-validation and external validation with partial least squares regression and (multiple) linear regression ${ }^{3}$

\begin{tabular}{|c|c|c|c|c|c|c|c|c|c|}
\hline \multirow[b]{2}{*}{ Model } & \multirow[b]{2}{*}{ Predictors used ${ }^{4}$} & \multicolumn{3}{|c|}{$\begin{array}{l}\text { Leave-one-out } \\
\text { cross-validation }\end{array}$} & \multicolumn{5}{|c|}{ External validation } \\
\hline & & Fac & RMSE & $\mathrm{r}$ & Fac & $\operatorname{Bias}(\mathrm{SE})^{1}$ & $\mathrm{~b}(\mathrm{SE})^{2}$ & RMSE & $\mathrm{r}$ \\
\hline $2^{3}$ & MY & - & 3.05 & 0.50 & - & $0.00(0.11)$ & $0.98(0.13)$ & 3.07 & 0.49 \\
\hline $3^{3}$ & MY, Mcont & - & 3.01 & 0.52 & - & $0.02(0.10)$ & $0.96(0.13)$ & 3.06 & 0.50 \\
\hline 4 & MIR & 6 & 3.15 & 0.45 & 6 & $0.01(0.11)$ & $0.88(0.16)$ & 3.27 & 0.38 \\
\hline $7^{3}$ & MY, BW & - & 2.95 & 0.55 & - & $0.00(0.10)$ & $0.99(0.12)$ & 2.97 & 0.54 \\
\hline 8 & MY, BW, MIR & 7 & 2.84 & 0.59 & 7 & $-0.02(0.10)$ & $0.90(0.11)$ & 2.98 & 0.54 \\
\hline
\end{tabular}

${ }^{1}$ Average difference between predicted values and true values in external validation data set.

${ }^{2}$ Linear regression coefficient of true value on predicted value.

${ }^{3}$ Linear regression model, no factors used.

${ }^{4} \mathrm{Mcont}=$ milk concentration (i.e., fat, protein, and lactose) $\mathrm{MY}=$ milk yield

in the present study therefore relate only to the analyses using the untreated spectra.

\section{PLS Regression}

The accuracy of predicting DMI and NEI from PLS regression is presented in Tables 4 and 5. The accuracy of prediction for the traits was dependent on the maximum number of explanatory factors permitted in the prediction equations. The greatest accuracy of predicting NEI when using only MIR spectral informa- tion in the leave-one-out cross-validation was achieved when the maximum number of permitted factors in the prediction equations was between 10 and 20 and in the external validation when the maximum number of permitted factors was between 12 and 20 (Figure 3). The PRESS statistic (Hastie et al., 2001) was used to determine the optimum number of permissible factors in the present study. The number of prediction factors used in the PLS models to achieve the greatest accuracy of prediction ranged from 5 to 8 depending on the trait and model (Tables 4 and 5). The $\mathrm{R}$ of predicting

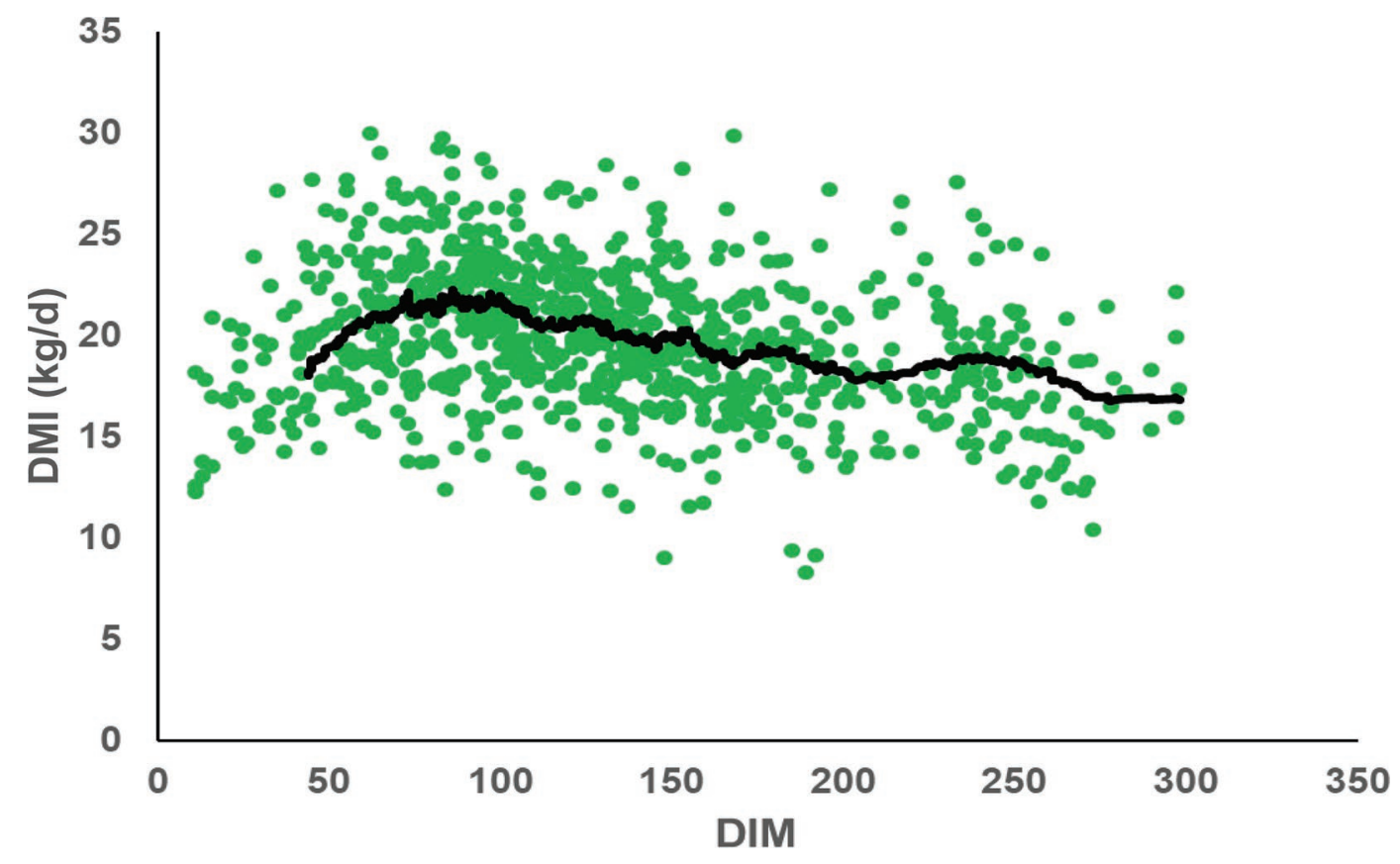

Figure 2. Lactation profile of the DMI on days with milk mid-infrared spectral recordings with an average trend line. Color version available online. 
Table 5. Average number of factors (Fac), root mean squared error (RMSE), correlation coefficient (r), mean bias (SE in parentheses), ${ }^{1}$ and slope (b; SE in parentheses) ${ }^{2}$ obtained from predicting net energy intake (NEI) using centered and scaled mid-infrared (MIR) spectra, tested using leave-one-out cross-validation and external validation using partial least squares regression and (multiple) linear regression ${ }^{3}$

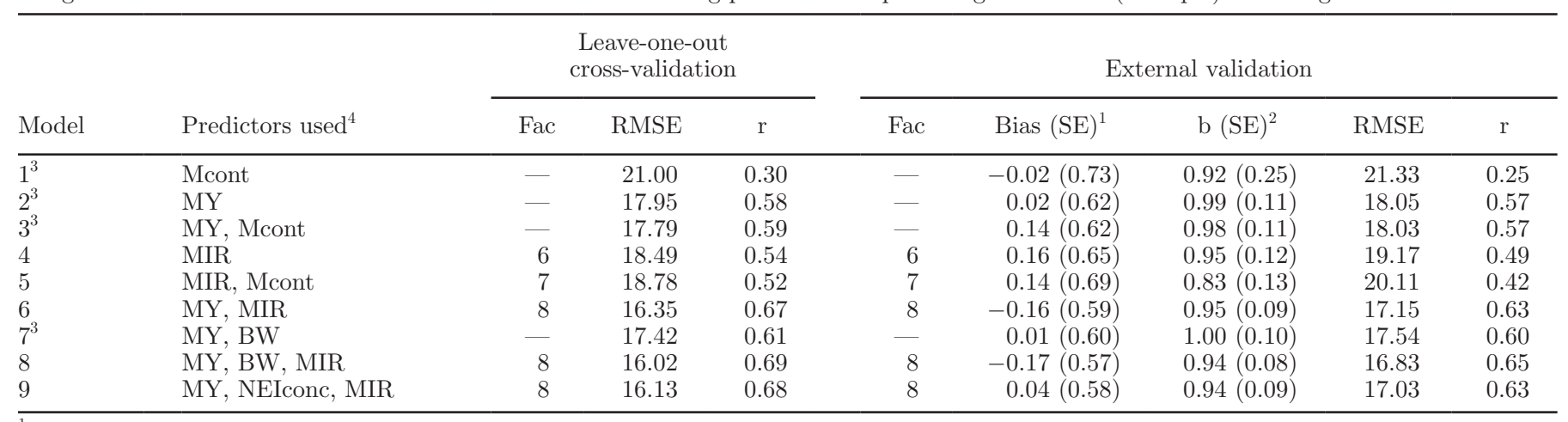

${ }^{1}$ Average difference between predicted values and true values in an external validation data set.

${ }^{2}$ Linear regression coefficient of true value on predicted values.

${ }^{3}$ Linear regression model, no factors used.

${ }^{4} \mathrm{Mcont}=$ milk concentration (i.e., fat, protein, and lactose); MY = milk yield; NEIconc $=$ energy intake from concentrate.

DMI and NEI was generally lower for external validation compared with cross-validation.

The $\mathrm{R}$ of prediction using external validation ranged from 0.24 to 0.54 for DMI and from 0.42 to 0.65 for NEI (i.e., models 4-6 and 8-9). Compared with the model with spectral information only (i.e., model 4 ), including MY in the prediction model increased the $\mathrm{R}$ of predicting DMI by 0.14 units in both the cross-validation and external validation and the R of NEI by between 0.13 (cross-validation) and 0.14 (external validation) units
(Tables 4 and 5). The greatest accuracy of predicting DMI (0.54) and NEI (0.65) in the external validation was achieved when both BW and MY were included in the model as predictors together with the MIR spectra (i.e., model 8).

For all the PLS models, the average LR coefficients of actual on predicted NEI and DMI in the external validation were not different from $1(P>0.05)$, which indicates that the predicted differences in NEI and DMI were close to the actual values (Tables 4 and 5).

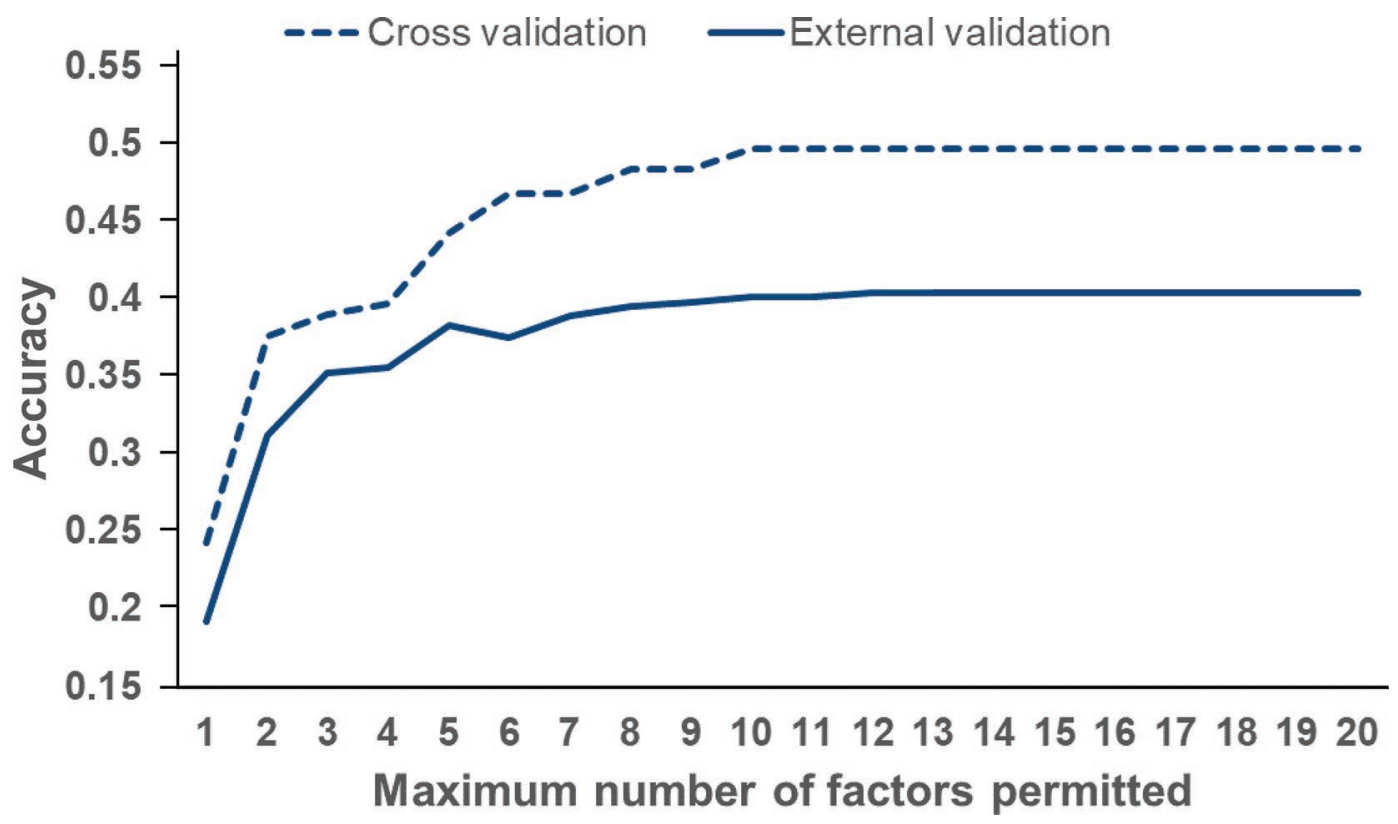

Figure 3. Influence of maximum number of predictive factors permitted in the calibration equation on the accuracy of the leave-one-out cross-validation and the external validation when predicting net energy intake using only milk mid-infrared spectral information. Color version available online. 
Table 6. Error variance/variance of the spectra $(\lambda)$, root mean squared error (RMSE), correlation coefficient $(\mathrm{r})$, mean bias (SE in parentheses), ${ }^{1}$ and slope (b; SE in parentheses) ${ }^{2}$ obtained from predicting DMI and net energy intake (NEI) using centered and scaled mid-infrared (MIR) spectra, tested using leave-one-out crossvalidation and external validation with best linear unbiased prediction ${ }^{3}$

\begin{tabular}{|c|c|c|c|c|c|c|c|}
\hline \multirow[b]{2}{*}{ Item $^{4}$} & \multirow[b]{2}{*}{$\lambda$} & \multicolumn{2}{|c|}{ Cross-validation } & \multicolumn{4}{|c|}{ External validation } \\
\hline & & RMSE & $\mathrm{r}$ & $\operatorname{Bias}(\mathrm{SE})^{1}$ & $\mathrm{~b}(\mathrm{SE})^{2}$ & RMSE & $\mathrm{r}$ \\
\hline \multicolumn{8}{|c|}{ DMI (kg/d) } \\
\hline Model 4 & 4 & 2.93 & 0.56 & $0.18(0.11)$ & $0.61(0.15)$ & 3.25 & 0.40 \\
\hline Model 5 & 20 & 2.96 & 0.54 & $0.47(0.12)$ & $0.50(0.16)$ & 5.99 & 0.31 \\
\hline Model 6 & 20 & 2.74 & 0.63 & $0.24(0.10)$ & $0.81(0.12)$ & 2.96 & 0.54 \\
\hline Model 8 & 20 & 2.96 & 0.54 & $0.49(0.12)$ & $0.49(0.16)$ & 3.40 & 0.30 \\
\hline \multicolumn{8}{|c|}{ NEI (MJ/d) } \\
\hline Model 4 & 4 & 17.10 & 0.63 & $0.95(0.65)$ & $0.66(0.16)$ & 19.14 & 0.50 \\
\hline Model 5 & 10 & 17.37 & 0.62 & $2.41(0.68)$ & $0.60(0.17)$ & 19.94 & 0.43 \\
\hline Model 6 & 10 & 15.77 & 0.70 & $1.08(0.59)$ & $0.89(0.12)$ & 17.18 & 0.63 \\
\hline Model 8 & 10 & 15.49 & 0.71 & $0.99(0.57)$ & $0.93(0.12)$ & 16.78 & 0.65 \\
\hline Model 9 & 4 & 15.43 & 0.71 & $1.02(0.57)$ & $0.90(0.12)$ & 16.78 & 0.65 \\
\hline
\end{tabular}

${ }^{1}$ Average difference between predicted values and true values in an external validation data set.

${ }^{2}$ Linear regression coefficient of true value on predicted value.

${ }^{3}$ In models 6, 8, and 9, milk yield, BW, and NEI from concentrate are treated as fixed effects.

${ }^{4}$ Model 4 used only MIR spectral information as a predictor. Model 5 used MIR spectral information together with fat, protein, and lactose concentration in milk as predictors. Model 6 used observations of milk yield together with MIR spectral information as predictors. Model 8 used observations of BW and milk yield together with MIR spectral information as predictors. Model 9 used observations of energy intake from concentrate and milk yield together with MIR spectral information as predictors.

The mean bias of prediction for NEI and DMI in the external validation was not different from $0(P>0.05)$, indicating unbiased prediction.

\section{BLUP}

The accuracy of BLUP predictions of DMI and NEI is presented in Table 6. The $\mathrm{R}$ of predicting DMI and NEI was lower in the external validation data set compared with the cross-validation data set. The $\mathrm{R}$ of prediction using the external validation ranged from 0.30 to 0.54 for DMI and from 0.43 to 0.65 for NEI. Compared with the model with spectral information only (i.e., model 4), including MY in the model (i.e., model 6) increased the R of predicting DMI by between 0.07 (cross-validation) and 0.14 (external validation) units and the R of predicting NEI by between 0.07 (cross-validation) and 0.13 (external validation) units (Table 6). Compared with all other BLUP models, using MIR spectra together with Mcont in the model (i.e., model 5) reduced the $\mathrm{R}$ of predicting $\mathrm{DMI}$ and NEI. The greatest accuracy for predicting DMI in the external validation (0.54) was achieved when predicted from the MIR spectra and MY combined (i.e., model 6). Treating BW as a fixed effect (i.e., model 8) when predicting DMI resulted in considerably less accuracy, especially in the external validation, than using models 4 and 6 . The greatest accuracy for predicting NEI in the external validation (0.65) was achieved when using a model including BW, MY, and MIR spectra (i.e., model 8) or using a model that included NEIconc, MY, and MIR spectra (i.e., model 9).

The LR coefficients of actual on predicted DMI for model 8 and the LR coefficients of actual on predicted NEI and DMI for models 4 and 5 in the external validation were less $(P<0.05)$ than 1 (Table 6$)$. For all the other models, the LR coefficient of actual on predicted NEI and DMI in the external validation was not different from $1(P>0.05)$. For DMI, only model 4 resulted in unbiased prediction $(P>0.05)$, whereas the mean bias of prediction was different from $0(P<$ $0.05)$ for all the other models. For NEI, only model 5 resulted in biased prediction $(P<0.05)$. Based on the regression coefficient and the bias, the best model for predicting NEI using BLUP included BW, MY, and the MIR spectra, and for DMI the best model included MY together with the MIR spectra.

\section{DISCUSSION}

The objective of the present study was to predict NEI and DMI as measures of feed intake in lactating Norwegian Red cows using milk MIR spectral data, sometimes accompanied in the prediction model by ancillary, often available, data. Accurate and easily accessible information about individual cow feed intake and efficiency could help manage feed costs and might enable genetic improvement of feed efficiency by providing large-scale data for breeding value evaluation. Wallén et al. (2017) showed that to genetically improve feed efficiency us- 
ing genomic selection, 4,000 additional genotyped and phenotyped heifers need to be added to the reference population annually. Generating such large quantities of feed intake measurements at a low cost is challenging. Hence, considerable research has focused on finding solutions to this conundrum, one of which is to pool data from different populations (de Haas et al., 2012; Veerkamp et al., 2013). Furthermore, McParland et al. (2011) proposed that MIR spectral data may be useful to predict energy intake because fat-to-protein ratio and milk fatty acid composition, which are reported to be associated with energy balance (Reist et al., 2002; Friggens et al., 2007), are already predicted from MIR spectra. McParland et al. (2011) successfully related milk MIR spectral data to both body energy status and energy intake in lactating Holstein dairy cows. The hypothesis, therefore, for the present study was that MIR spectral data could be used as a predictor of NEI and DMI. Of particular interest was the marginal improvement in predictive ability by considering data from potentially informative traits often available at the time of milk sampling but also the usefulness of BLUP approaches in improving the predictive ability over the commonly used PLS approaches. Moreover, studies to date have been confined to Holstein-Friesian cows, but Norwegian Red cows predominate in Norway, and the ability of milk MIR to predict intake in this breed is currently unknown.

\section{Prediction Equations}

The number of explanatory factors permitted in the prediction model affected the resulting prediction accuracy in the present study. This is in line with the results from both McParland et al. (2011), who predicted body energy status in Holstein cows using PLS on milk MIR, and Martínez et al. (2017), who studied a model selection criterion for PLS regression using simulations. In the present study, too few explanatory factors in the model contributed to a reduction in $\mathrm{R}$, most likely due to an oversimplified prediction model. The optimum number of permissible factors in the present study appears to be 10 to 12 when predicting NEI. The optimum number of permissible factors in the study of McParland et al. (2011) was 20 for body energy status when predictions were across lactation. For this reason, the maximum number of explanatory factors in the prediction models was limited to 12 in the present study, which was lower than the 20 explanatory factors permitted in the study of McParland et al. (2011) and is lower than the 16 factors permitted by Soyeurt et al. (2011), who also used PLS but in the prediction of milk fatty acids from milk MIR in dairy cows.
The results from the present study regarding smoothing of the spectral data agree with those of McParland et al. (2011), who used MIR of milk to predict animallevel phenotypes, yet contradict those of Soyeurt et al. (2011), who predicted milk fatty acid composition from milk MIR. The apparent discrepancy between studies could simply be due to whether the MIR data originated from 1 or multiple spectrometers. Soyeurt et al. (2011) used data from multiple spectrometers, whereas data from only 1 spectrometer was used both by McParland et al. (2011) and in the present study.

\section{Comparison Between PLS and BLUP Methods}

Partial least squares methods traditionally have been used to develop the prediction equations in dairy cows from milk MIR (McParland et al., 2011, 2012, 2014; De Marchi et al., 2014). Best linear unbiased prediction approaches, however, are the norm in genetic and genomic evaluations. Because one of the objectives of the present study was to develop prediction models for possible use in breeding programs, consideration was given to whether BLUP approaches could also be used to predict NEI and DMI, thus simplifying the pipelines used. Furthermore, because BLUP provides the opportunity to treat some of the predictors as fixed effects (i.e., MY and BW) and because there is strong prior knowledge to indicate these are informative in predicting feed intake (Berry and Crowley, 2013; McParland et al., 2014), we expected that BLUP could improve prediction accuracy relative to PLS. Although the R of cross-validation was generally better for BLUP, the $\mathrm{R}$ of both BLUP and PLS was similar in the external validation (Tables 4, 5, and 6). Worrying, however, was the evidence of mean bias and an LR coefficient of the actual values on BLUP-predicted values differing from unity; such biases were not evident for PLS. McParland et al. (2011) reported a LR coefficient of the actual values of energy balance on PLS-predicted values not differing from unity. However, the LR coefficient of actual on predicted energy content was different from unity (0.77-0.83 \pm 0.06$)$. McParland et al. (2011) also reported a biased prediction for energy content. Mean biases, however, are not necessarily a big issue for genetic evaluations becase genetic evaluations are all undertaken within a contemporary group and in doing so remove the mean contemporary group effect from the individual records. One possible reason why BLUP resulted in biased predictions is that $\lambda$ was chosen based on external validation $R$ values and was not estimated using variance components. Thus, based on our results, PLS seems to be a better method for predicting feed intake using milk MIR than BLUP. 


\section{Improving Feed Efficiency}

In the present study, the most accurate model for predicting both DMI and NEI included BW, MY, and MIR spectra when using PLS. For DMI, including only MY and BW in the prediction model resulted in similar accuracy. Even though BW itself is currently not readily available on many commercial dairy farms, it can be estimated using, for example, chest width because a strong genetic correlation between BW and chest width has been reported (0.75-0.86; Veerkamp and Brotherstone, 1997). Moreover, developments in sensor technologies could result in live weight (and other phenotypes such as BCS) being routinely recorded daily in the future. Using Mcont in the prediction model or combining them with MIR spectra resulted in low accuracy of prediction. However, combining MY with Mcont increased the accuracy considerably. It is clear that using only Mcont or MIR spectra in the model does not result in high prediction accuracy for NEI or DMI; in fact, the accuracy of prediction using just the MIR data was always inferior to a model that used just MY, fat, protein, and lactose concentration. The use of MY and BW together with MIR spectra in the prediction model increased the prediction accuracy considerably and resulted in an R of 0.65 for NEI in the external validation. This is slightly lower than the study of McParland et al. (2014), where PLS was used to predict effective energy intake using just MY and milk MIR, resulting in an $\mathrm{R}$ of 0.70 in the external validation. The differences in the results between McParland et al. (2014) and the present study could be due to the fact that the data used in the present study were considerably smaller than those used in the study of McParland et al. (2014), which might have an effect on the robustness of the prediction models in the present study. Furthermore, the variability in the frequency of data available as well as the diversity in feeding experiments represented in the data set used in the present study may also have affected the results of the present study. Nonetheless, most of the data used in the present study originated from a feeding experiment with regular collection of data. Additionally, the construction of the calibration and validation data sets differed between the study of McParland et al. (2014) and the present study. Finally, the use of different wavelengths from the MIR spectra in the development of the prediction models across studies could have an effect on the prediction accuracies.

Dry matter intake was more difficult to predict using milk MIR in the present study because the spectra are expected to reflect the absorbed feed components and therefore cannot necessarily predict the amount of feed that the cow ate to obtain these absorbed feed components. Therefore, NEI is more useful for practical application because it reflects the energy in the consumed feed instead of merely the amount of feed consumed. The prediction accuracy of 0.65 (external validation) for NEI seems promising, especially if we consider that energy intake is a phenotype that itself encompasses recording errors (McParland and Berry, 2016). For instance, diurnal variation may also exist in energy intake (McParland et al., 2011), which makes the maximum achievable prediction accuracy less than $100 \%$ (possibly $75 \%$ for body energy status according to McParland et al., 2011, and $70 \%$ for energy intake according to McParland et al., 2014). McParland et al. (2015) reported heritable genetic variability for energy intake, energy balance, and residual energy intake predicted using milk MIR. In addition, this variability is sufficiently large to justify consideration of including FE in dairy cattle breeding goals (Hurley et al., 2017). McParland et al. (2015) also reported that phenotypic differences in energy intake existed among animals, which were stratified, based on their EBV for energy intake predicted from MIR. Therefore, genetic improvement of feed efficiency could be possible. Feed intake itself is the actual gold standard trait and can be used when actual feed intake data are available. Hence, as more actual feed intake data accumulate for an animal or a sire, the importance of the MIR-predicted feed intake diminishes. Nonetheless, the accuracy of prediction of feed intake from daughter phenotypes can never be greater than the genetic correlation between the predicted phenotypes and the actual feed intake.

Results from the present study suggest that MIR spectral data can be used to predict NEI as a measure of feed intake in Norwegian Red dairy cattle. When using PLS with 8 factors, the most accurate model of predicting NEI included all of BW, MY, and MIR spectra as predictors. The BLUP predictions yielded similar accuracies but were biased. Nonetheless, no matter which feed efficiency measure would be chosen, before including the trait in the breeding program, genetic correlations between predicted feed intake, actual feed intake, and other performance traits - especially health and fertility traits - need to be estimated to derive the selection index weights.

\section{ACKNOWLEDGMENTS}

The helpful comments of 3 anonymous reviewers are gratefully acknowledged. The authors acknowledge the Norwegian Research Council (Lysaker, Norway; project no. 225233/E40), the breeding and AI organization GENO (Ås, Norway), and the dairy foods company TINE (Oslo, Norway) for funding. Thanks to Ingunn Schei (Department of Animal and Aquacultural Sci- 
ences, Norwegian University of Life Sciences, Ås, Norway) for help with the NorFor calculations and to the Norwegian University of Life Sciences research farm (Ås, Norway) and the Norwegian Dairy Herd Recording System (Ås, Norway) for providing the data. Cubic splines and MATLAB computations were performed at the Abel cluster at the University of Oslo.

\section{REFERENCES}

Berry, D. P. 2015. Breeding the dairy cow of the future: What do we need? Anim. Prod. Sci. 55:823-837.

Berry, D. P., and J. J. Crowley. 2013. Cell Biology Symposium: Genetics of feed efficiency in dairy and beef cattle. J. Anim. Sci 91:1594-1613

de Haas, Y., M. P. L. Calus, R. F. Veerkamp, E. Wall, M. P. Coffey, H. D. Daetwyler, B. J. Hayes, and J. E. Pryce. 2012. Improved accuracy of genomic prediction for dry matter intake of dairy cattle from combined European and Australian datasets. J. Dairy Sci 95:6103-6112.

De Marchi, M., V. Toffanin, M. Cassandro, and M. Penasa. 2014 Invited review: Mid-infrared spectroscopy as phenotyping tool for milk traits. J. Dairy Sci. 97:1171-1186.

Ferragina, A., G. de los Campos, A. I. Vazquez, A. Cecchinato, and G. Bittante. 2015. Bayesian regression models outperform partial least squares methods for predicting milk components and technological properties using infrared spectral data. J. Dairy Sci. 98:8133-8151.

Friggens, N. C., C. Ridder, and P. Løvendahl. 2007. On the use of milk composition measures to predict energy balance of dairy cows. J. Dairy Sci. 90:5453-5467.

Hastie, T., R. Tibshirani, and J. H. Friedman. 2001. The Elements of Statistical Learning: Data Mining, Inference, and Prediction. 1st ed. Springer, New York, NY.

Hurley, A. M., N. López-Villalobos, S. McParland, E. Lewis, E. Kennedy, M. O'Donovan, J. L. Burke, and D. P. Berry. 2017. Genetics of alternative definitions of feed efficiency in grazing lactating dairy cows. J. Dairy Sci. 100:5501-5514.

Martínez, J. L., H. Saulo, H. B. Escobar, and J. Leao. 2017. A new model selection criterion for partial least squares regression. Chemom. Intell. Lab. Syst. 169:64-78. https://doi.org/10.1016/j chemolab.2017.08.006

McParland, S., G. Banos, B. McCarthy, E. Lewis, M. P. Coffey, B O'Neill, M. O'Donovan, E. Wall, and D. P. Berry. 2012. Validation of mid-infrared spectrometry in milk for predicting body energy status in Holstein-Friesian cows. J. Dairy Sci. 95:7225-7235.

McParland, S., G. Banos, E. Wall, M. P. Coffey, H. Soyeurt, R. F. Veerkamp, and D. P. Berry. 2011. The use of mid-infrared spectrometry to predict body energy status of Holstein cows. J. Dairy Sci. 94:3651-3661.

McParland, S., and D. P. Berry. 2016. The potential of Fourier transform infrared spectroscopy of milk samples to predict energy intake and efficiency in dairy cows. J. Dairy Sci. 99:4056-4070.
McParland, S., E. Kennedy, E. Lewis, S. G. Moore, B. McCarthy, M. O'Donovan, and D. P. Berry. 2015. Genetic parameters of dairy cow energy intake and body energy status predicted using midinfrared spectrometry of milk. J. Dairy Sci. 98:1310-1320.

McParland, S., E. Lewis, E. Kennedy, S. G. Moore, B. McCarthy, M. O'Donovan, S. T. Butler, J. E. Pryce, and D. P. Berry. 2014 Mid-infrared spectrometry of milk as a predictor of energy intake and efficiency in lactating dairy cows. J. Dairy Sci. 97:5863-5871.

Meuwissen, T. H. E., B. J. Hayes, and M. E. Goddard. 2001. Prediction of total genetic value using genome-wide dense marker maps. Genetics 157:1819-1829.

Miglior, F., B. L. Muir, and B. J. Van Doormaal. 2005. Selection indices in Holstein cattle of various countries. J. Dairy Sci. 88:12551263

Philipsson, J., and B. Lindhé. 2003. Experiences of including reproduction and health traits in Scandinavian dairy cattle breeding programmes. Livest. Prod. Sci. 83:99-112.

Reist, M., D. Erdin, D. Von Euw, K. Tschuemperlin, H. Leuenberger, Y. Chilliard, H. M. Hammon, C. Morel, C. Philipona, Y. Zbinden, N. Kuenzi, and J. W. Blum. 2002. Estimation of energy balance at the individual and herd level using blood and milk traits in highyielding dairy cows. J. Dairy Sci. 85:3314-3327.

Shalloo, L., P. Dillon, M. Rath, and M. Wallace. 2004. Description and validation of the Moorepark dairy system model. J. Dairy Sci. $87: 1945-1959$

Soyeurt, H., F. Dehareng, N. Gengler, S. McParland, E. Wall, D. P Berry, M. Coffey, and P. Dardenne. 2011. Mid-infrared prediction of bovine milk fatty acids across multiple breeds, production systems, and countries. J. Dairy Sci. 94:1657-1667.

Svendsen, M., P. Skipenes, and I. L. Mao. 1993. Genetic parameters in the feed conversion complex of primiparous cows in the first two trimesters. J. Anim. Sci. 71:1721-1729.

Van Es, A. J. H. 1975. Feed evaluation for dairy cows. Livest. Prod. Sci. 2:95-107.

Van Es, A. J. H. 1978. Feed evaluation for ruminants. 1. The systems in use from May 1977-onwards in the Netherlands. Livest. Prod. Sci. 5:331-345.

Veerkamp, R. F., and S. Brotherstone. 1997. Genetic correlations between linear type traits, food intake, live weight and condition score in Holstein Friesian dairy cattle. Anim. Sci. 64:385-392.

Veerkamp, R. F., J. E. Pryce, D. Spurlock, D. Berry, M. Coffey, P. Løvendahl, R. van der Linde, J. Bryant, F. Miglior, Z. Wang, M. Winters, N. Krattenmacher, N. Charfeddine, J. Pedersen, and Y. de Haas. 2013. Selection on feed intake or feed efficiency: A position paper from gDMI breeding goal discussions. Pages 15-22 in Proc. Interbull Ann. Mtg., Nantes, France. Interbull No. 47. Interbull, Uppsala, Sweden.

Volden, H. 2011. NorFor-The Nordic feed evaluation system. European Federation of Animal Sciences No. 130. Wageningen Academic Publishers, Wageningen, the Netherlands.

Wallén, S. E., M. Lillehammer, and T. H. E. Meuwissen. 2017. Strategies for implementing genomic selection for feed efficiency in dairy cattle breeding schemes. J. Dairy Sci. 100:6327-6336.

Zimmermann, B., and A. Kohler. 2013. Optimizing Savitzky-Golay parameters for improving spectral resolution and quantification in infrared spectroscopy. Appl. Spectrosc. 67:892-902. 


\section{APPENDIX}

Formulae used to calculate energy intake based on NorFor (Volden, 2011).

\section{Gross Energy Intake}

GEI $=$

$\left\{\begin{array}{l}24.1 \times \sum_{\mathrm{i}}\left(\mathrm{DMI}_{\mathrm{i}} \times \mathrm{CPCorr}_{\mathrm{i}}\right)+36.6 \\ \times \sum_{\mathrm{i}}\left(\mathrm{DMI}_{\mathrm{i}} \times \mathrm{CFat}_{\mathrm{i}}\right)+18.5 \\ \times \sum_{\mathrm{i}}\left[\mathrm{DMI}_{\mathrm{i}} \times\left(\mathrm{OM}_{\mathrm{i}}-\mathrm{CPCorr}_{\mathrm{i}}-\mathrm{CFat}_{\mathrm{i}}-\frac{\mathrm{CP}_{\mathrm{i}}}{6.25} \times \frac{\mathrm{NH}_{3} \mathrm{~N}_{\mathrm{i}}}{1,000}\right)\right]\end{array}\right\}$ $/ 1,000$,

where gross energy intake (GEI) is expressed as megajoules per day and, for feedstuff $\mathrm{i}, \mathrm{DMI}_{\mathrm{i}}$ is the DMI $(\mathrm{kg} / \mathrm{d})$, CPcorr $_{\mathrm{i}}$ is the content of ammonia- or ureacorrected $\mathrm{CP}, \mathrm{CFat}_{\mathrm{i}}$ is the crude fat content $(\mathrm{g} / \mathrm{kg}$ of $\mathrm{DM}), \mathrm{OM}_{\mathrm{i}}$ is the $\mathrm{OM}$ content $(\mathrm{g} / \mathrm{kg}$ of $\mathrm{DM}), \mathrm{CP}_{\mathrm{i}}$ is the $\mathrm{CP}$ content $(\mathrm{g} / \mathrm{kg}$ of $\mathrm{DM})$, and $\mathrm{NH}_{3} \mathrm{~N}_{\mathrm{i}}$ is the ammonia or urea $\mathrm{N}$ content $(\mathrm{g} / \mathrm{kg}$ of $\mathrm{CP} ; \mathrm{i}=1, \ldots, \mathrm{n})$.

\section{ME Intake}

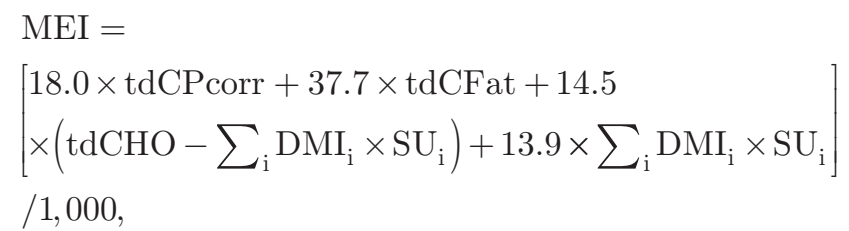

where ME intake (MEI) is expressed as megajoules per day, $\mathrm{DMI}_{\mathrm{i}}$ is the DMI $(\mathrm{kg} / \mathrm{d})$ of feedstuff $\mathrm{i}(\mathrm{i}=1, \ldots$, $\mathrm{n}), \mathrm{SU}_{\mathrm{i}}$ is the sugar content $(\mathrm{g} / \mathrm{kg}$ of $\mathrm{DM})$ of feedstuff $\mathrm{i}(\mathrm{i}=1, \ldots, \mathrm{n})$, tdCPcorr is the total-tract digestion of ammonia- or urea-corrected $\mathrm{CP}$, tdCFat is the total-tract digestion of crude fat, and tdCHO is the total-tract digestion of carbohydrates.

NEI

In NorFor, NEI is based on the equations of Van Es (1975, 1978). The NEI per individual was calculated based on ME and gross energy of feed:

$$
\mathrm{NEI}=0.6 \times[1+0.004 \times(\mathrm{q}-57)] \times \mathrm{MEI},
$$

where NEI is expressed as megajoules per day and $q$ is the ratio $(\%)$ between $\mathrm{ME}$ and gross energy. 